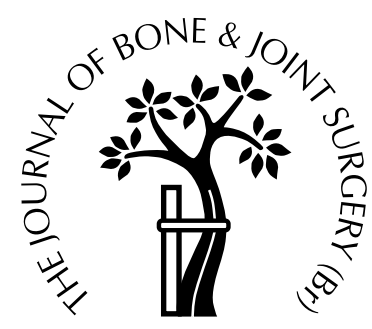

\title{
Factors which affect the progression of osteoarthritis after rotational acetabular osteotomy
}

\author{
H. Koga, M. Matsubara, K. Suzuki, S. Morita, T. Muneta \\ From the Tokyo Medical and Dental University Hospital, Tokyo, Japan
}

W e have investigated the factors which affect the progression of osteoarthritis after rotational acetabular osteotomy (RAO).

Between 1984 and 1998, we treated 60 dysplastic hips by RAO. The mean age at surgery was 31.6 years (13 to 51) and the mean period of follow-up was 4.6 years ( 2 to 9.5). The thickness of the articular cartilage on the weight-bearing area, pre- and postoperative acetabular cover, and the sphericity of the femoral head were used for radiological assessment.

The osteoarthritis did not progress in 39 hips. Significant factors which affected the radiological grade included sphericity of the femoral head and the postoperative acetabular cover. The surgical approach and preoperative acetabular cover did not affect the progression of osteoarthritis. Patients were divided into two groups according to the surgical approach used, either conventional ( 23 hips) or modified (37 hips). Significant factors included the postoperative acetabular cover in the modified approach, and the sphericity of the femoral head in the conventional approach.

It is critical that the postoperative cover is sufficient, especially when RAO is carried out using our modified technique.

J Bone Joint Surg [Br] 2003;85-B:963-8. Received 16 April 2002; Accepted 22 April 2003

Rotational acetabular osteotomy (RAO) is an effective treatment for young adults with early osteoarthritis (OA) secondary to hip dysplasia because it makes long-term joint

H. Koga, MD, Orthopaedic Surgeon

M. Matsubara, MD, Orthopaedic Surgeon

K. Suzuki, MD, Orthopaedic Surgeon

S. Morita, MD, Assistant Professor

T. Muneta, MD, Professor

Department of Orthopaedic Surgery, Tokyo Medical and Dental University

Hospital, 1-5-45 Yushima, Bunkyo, Tokyo 113-0034, Japan.

Correspondence should be sent to Dr H. Koga.

C 2003 British Editorial Society of Bone and Joint Surgery doi:10.1302/0301-620X.85B7.13460 \$2.00 preservation possible. ${ }^{1-5}$ Some hips, however, show rapid progression of OA with thinning of the articular cartilage of the joint or collapse of the acetabulum a few years after surgery. We have investigated the factors which affect the progression of OA after RAO.

\section{Patients and Methods}

Between October 1984 and July 1998, RAO was undertaken on 60 hips in 57 young adult patients with early-stage OA secondary to hip dysplasia. There were eight men (nine hips) and 49 women (51 hips). The mean age at the time of surgery was 31.6 years (13 to 51). Radiographs taken before operation showed an improved congruity between the femoral head and the acetabulum in $20^{\circ}$ of abduction and $0^{\circ}$ to $30^{\circ}$ of anterior inclination of the pelvis (RAO position). Three patients had bilateral procedures. The main indication for surgery was pain. The mean period of follow-up was 4.6 years (2 to 9.5).

The operative technique has been described in many reports, but we used a modified method in the last 37 hips. ${ }^{1-5}$ This was a lateral transtrochanteric approach through a single lateral incision in order to expose the entire circumference of the acetabulum. We next detached the greater trochanter pericapsularly from the femur with gluteus medius and gluteus minimus, in order to preserve the strength of the hip abductors. ${ }^{6}$ In the first 23 hips we used the original approach as described by Ninomiya and Tagawa. ${ }^{2}$ This was a combination of anterior iliofemoral and posterior approaches through a single anterior, convex incision. The transferred acetabulum was cut to approximately $20 \mathrm{~mm}$ in thickness and stabilised into the bony pelvis by Kirschner wires in the first 44 hips and by bioabsorbable screws of 4.5 $\mathrm{mm}$ in diameter in the last 16 hips.

Patients rested in bed for a week after surgery. Active exercises began in the second week with partial weightbearing, and the patients were discharged using one or two crutches six weeks after surgery. These were usually discarded three months after surgery. The goal was a range of movement which was identical to the preoperative range.

Anteroposterior radiographs of the pelvis were taken before and after the operation. We compared the most recent radiographs with those taken immediately after surgery. The following signs were used to assess progression of 


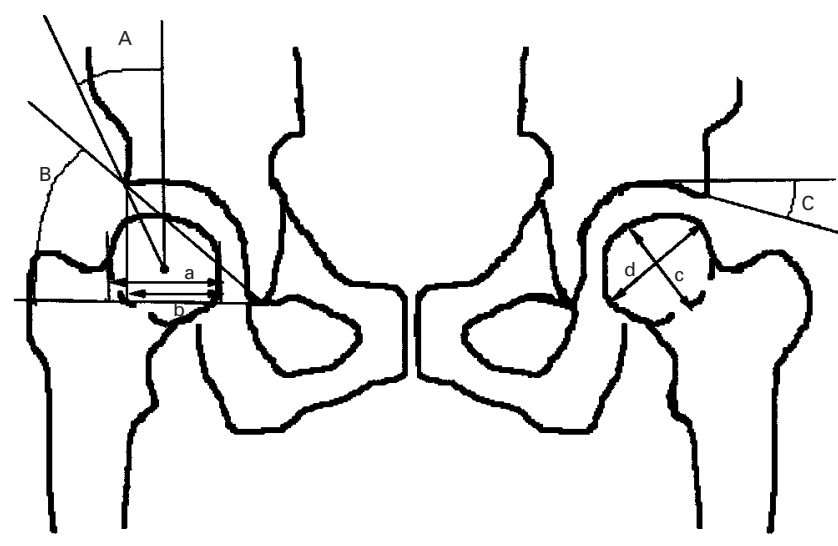

Fig. 1

Diagram showing the indices of acetabular cover and femoral head sphericity. (A, CE angle; B, sharp angle; C, acetabular edge angle (AEA); b/a, acetabular head index (AHI); d/c, sphericity of the femoral head; d/c $<1.2$ indicates a spherical head; and $\mathrm{d} / \mathrm{c}>1.2$ indicates a deformed head.)

OA: 1) diminution of articular cartilage $(\geq 1 \mathrm{~mm})$ in the weight-bearing areas; and 2) collapse of the transferred

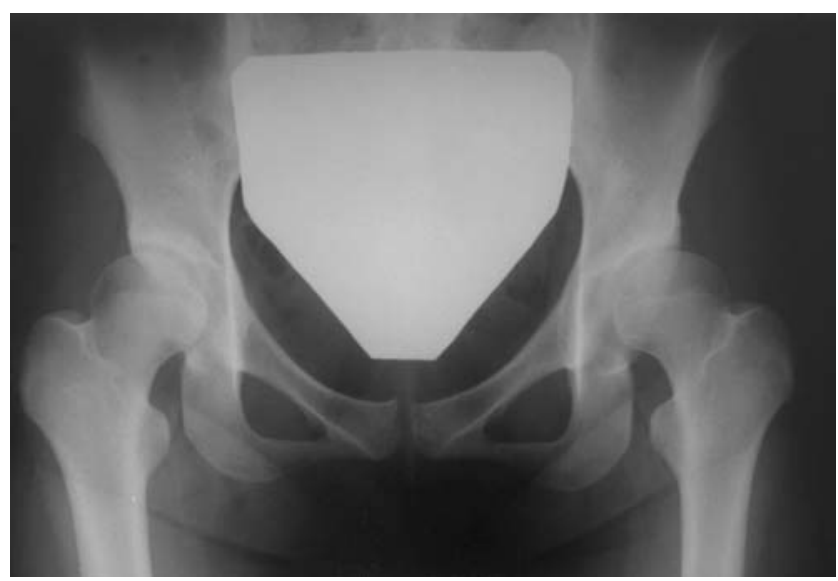

Fig. 2a

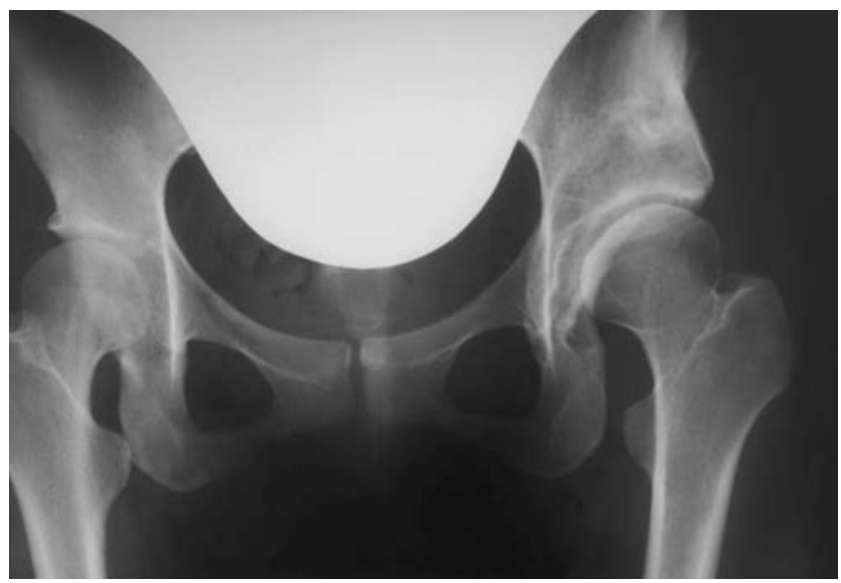

Fig. 2c acetabulum. Pre- and postoperative acetabular cover and the preoperative sphericity of the femoral head were also used for radiological assessment. The pre- and postoperative centre-edge (CE) angle, the Sharp angle, the acetabular edge angle (AEA), and acetabular head index (AHI) were used as indices of cover. Sphericity of the femoral head was assessed by measuring the ratio of the major and minor axes on the preoperative radiographs. ${ }^{7}$ A value of $<1.2$ indicated a spherical head and of $\geq 1.2$, a deformed head (Fig. 1).

Statistical analysis. We investigated statistically significant factors (Mann-Whitney U-test, $\mathrm{p}<0.05$ ) which could affect the progression of OA after RAO by comparing patients with good outcomes with those with poor outcomes using the signs indicating progression of OA, age at the time of surgery and the surgical approach. We also divided the patients into two groups by surgical approach and the same statistical analyses were undertaken.

\section{Results}

No patient had severe complications such as nerve injury, thromboembolism, or deep infection. All had a well transferred acetabulum.

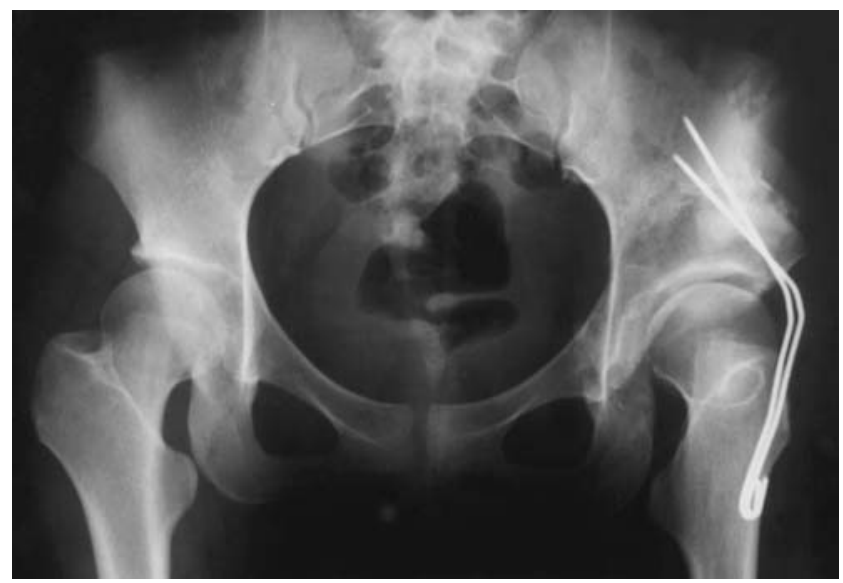

Fig. $2 b$

Radiographs of a 21-year-old woman with early OA of the left hip, showing a) preoperative acetabular dysplasia; b) using the original approach the transferred acetabulum stabilised by Kirschner wires; and c) 5.5 years later, excellent remodelling of the transferred acetabulum and no thinning of articular cartilage. 


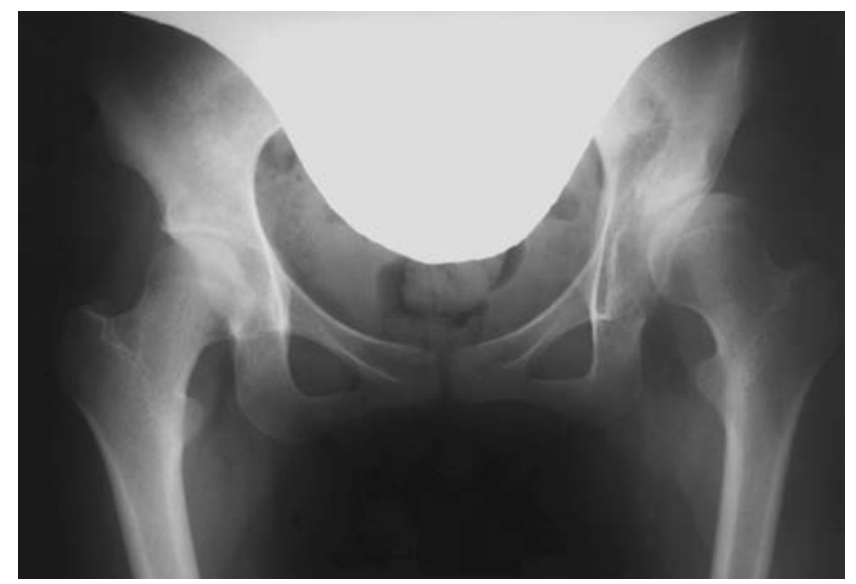

Fig. 3a

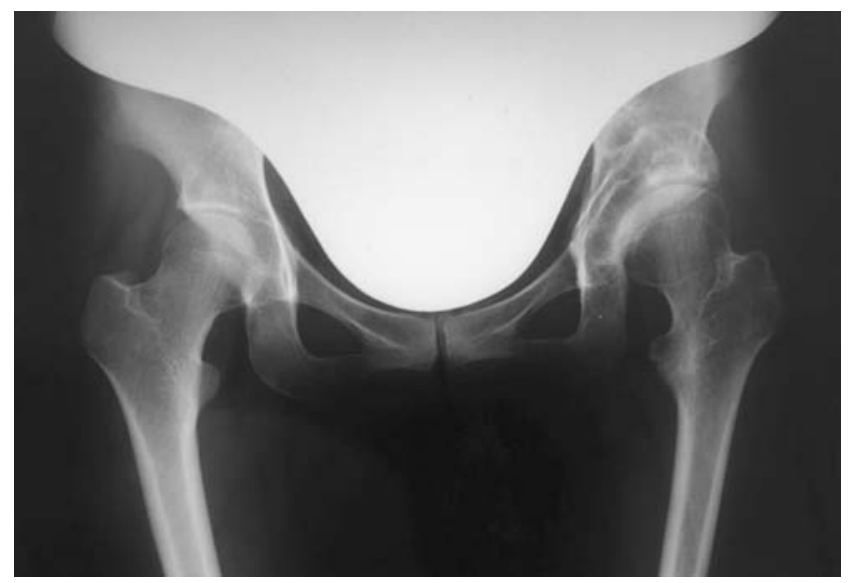

Fig. 3c

Radiologically, a good outcome was seen in 39 hips $(65 \%)$, but progression of OA, indicating a poor outcome, occurred in $21(35 \%)$. We compared the 39 hips with a good outcome (Figs 2 and 3) with the 21 with a poor outcome (Fig. 4).

The CE angle improved from a mean of $0.4^{\circ}$ to a mean of $32.4^{\circ}$ in the good group and from $-3.7^{\circ}$ to $29.1^{\circ}$ in the poor group. The Sharp angle improved from $51.7^{\circ}$ to $40.3^{\circ}$ in the good group and from $52.0^{\circ}$ to $40.7^{\circ}$ in the poor group. Before operation the AEA in the good group was less than $0^{\circ}$ in 27 hips, $0^{\circ}$ or more in 12 , but after operation it was $0^{\circ}$ or more in all hips. Similarly, the AEA in the poor group was $<0^{\circ}$ in 16 hips, $\geq 0^{\circ}$ in five hips, and postoperatively improved to $<0^{\circ}$ in four hips. The AHI improved from 0.540 to 0.863 in the good group and from 0.497 to 0.807 in the poor group. The acetabular cover improved in both groups with no significant difference between them. In the good group, 26 femoral heads were spherical and 13 deformed, while in the poor group, eight were spherical and 13 deformed. The mean age at the time of surgery was 31.1 years in the good group and 32.5 years in the poor group. We used the modified approach in 25 of 39 patients in the good group and in 12 of 19 patients in the poor group.

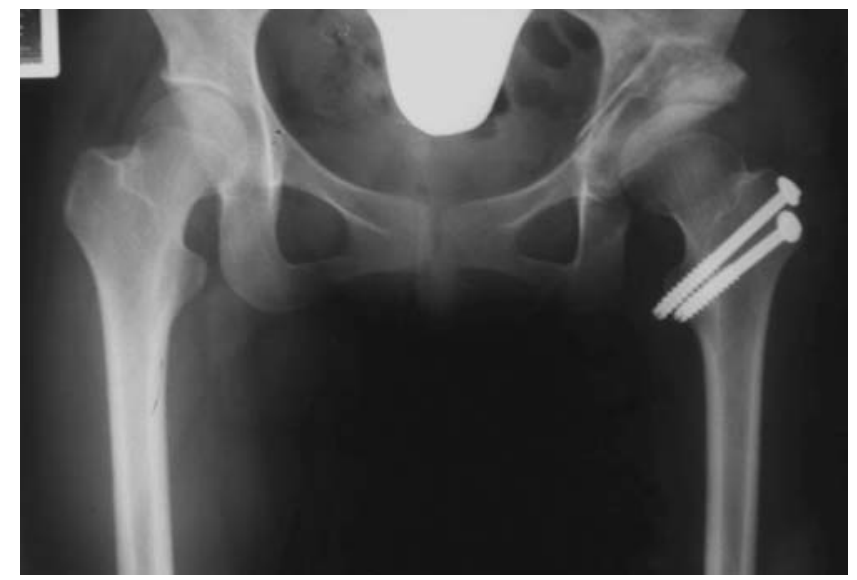

Fig. $3 b$

Radiographs of a 20-year-old woman with early OA of the left hip, showing a) preoperative severe dysplasia; b) using our modified approach, good cover by the transferred acetabulum which was stabilised by $4.5 \mathrm{~mm}$ diameter bioabsorbable screws; and c) 2.5 years later, no progression of OA.

As a result of analysing these data, we identified significant risk factors. These were: 1) a postoperative $\mathrm{CE}$ angle of $\left.<25^{\circ}, 2\right)$ a postoperative AEA of $<0^{\circ}, 3$ ) a postoperative AHI of $<0.8,4$ ) patients who were 25 years or older at the time of surgery, and 5) a deformed femoral head (Table I). The factors which affect the progression of OA therefore include sphericity of the femoral head and the postoperative acetabular cover. The surgical approach and preoperative acetabular cover did not appear to influence the progression of the OA. When the results were divided by surgical approach and analysed, we found the significant factors to be: 1) the postoperative $\mathrm{CE}$ angle; 2) the postoperative Sharp angle; 3 ) the postoperative AEA; and 4) the postoperative AHI in the modified approach. Sphericity of the femoral head and the postoperative AEA were included in the conventional approach (Table I).

\section{Discussion}

We carried out RAO on patients less than 50 years of age with early OA secondary to hip dysplasia when improved congruity between the femoral head and acetabulum was obtained radiographically in the RAO position. These 


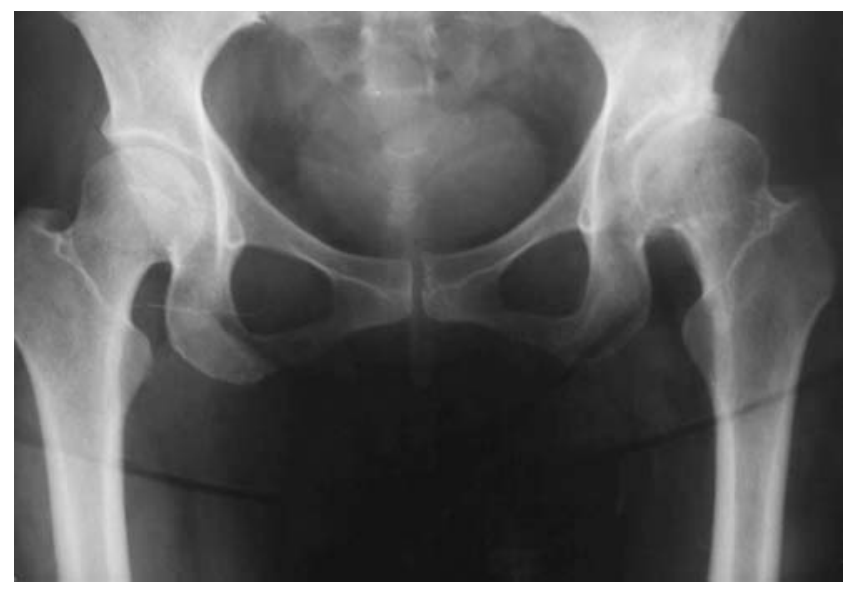

Fig. 4a

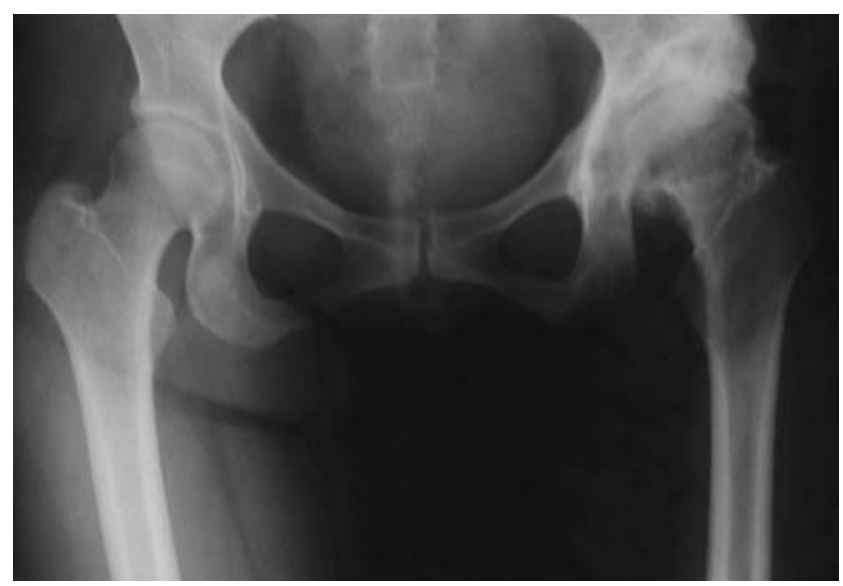

Fig. 4c

results, however, show that there are risk factors which may affect the progression of OA.

The main radiographic finding is narrowing of the jointspace indicative of thinning of articular cartilage and progression of OA. One of the reasons for undertaking RAO is that the articular cartilage of the acetabulum in the RAO position is preserved to some degree. There are some hips in which the articular cartilage thins and OA progresses rapidly after surgery. Ninomiya ${ }^{3}$ reported that collapse of the acetabulum could occur when the osteotomised segment was too thin. Wagner ${ }^{8}$ stated that care should be taken to keep the osteotomy at least $10 \mathrm{~mm}$ from the acetabular roof in order to avoid trophic disturbance of the acetabulum. Matsui et $\mathrm{al}^{9}$ reported that chondrolysis of the hip and necrosis of the transferred acetabulum after RAO may result from trophic disturbance caused by the combination of a thin transferred acetabulum and the transtrochanteric approach. This may impair the circulation around the acetabulum and hip, particularly to the capsule. A thin osteotomy impairs circulation and can cause necrosis of the subchondral lamellar bone plate. Pressure from the femoral head subsequently causes collapse of the acetabulum. In view of these reports, we kept the osteot-

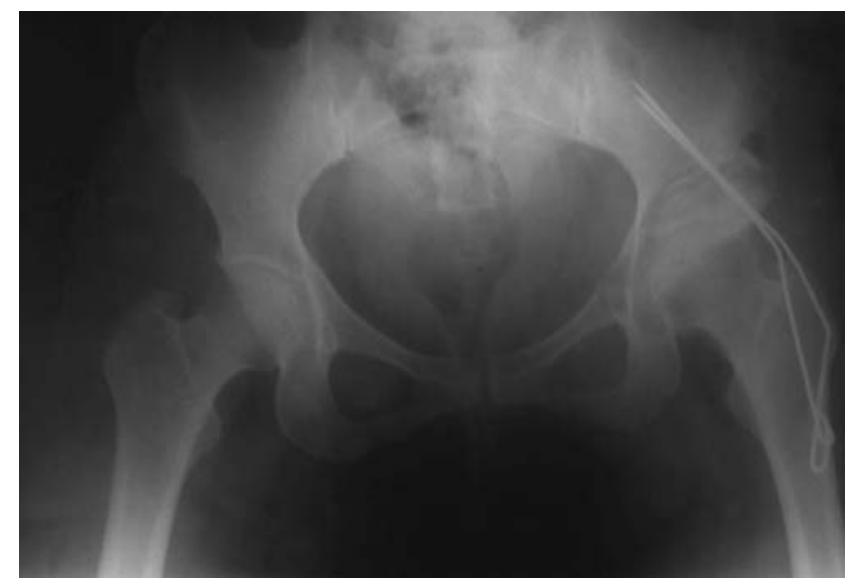

Fig. $4 b$

Radiographs of a 41-year-old woman with OA of the left hip, showing a) preoperative OA secondary to acetabular dysplasia; $b$ ) the operation was performed using the original approach; and c) six years later, marked narrowing of the joint space.

omy approximately $20 \mathrm{~mm}$ thick in all hips. Otherwise, there was no significant difference between the original and the modified approaches. This shows that exposure of the entire circumference of the acetabulum, and detachment of the greater trochanter in the lateral transtrochanteric approach, do not always impair circulation around the hip.

The aim of RAO is to expand the weight-bearing area in order to diminish the load per area in dysplastic hips. Our results showed significant differences in the postoperative CE angle, AEA and AHI, although we saw no correlation with the preoperative data. This suggests that postoperative grades are not influenced by preoperative ones, but are affected by postoperative acetabular cover. This emphasises that care should be taken to transfer the acetabulum sufficiently and to make full use of the available cover for each patient. RAO is also indicated in severe dysplasia because preoperative cover does not influence the progression of OA. RAO may not be suitable for patients whose femoral heads are deformed since the sphericity of a femoral head influences the progression of OA. Improvement of congruity cannot be obtained with a deformed femoral head even if the acetabulum is transferred sufficiently. 
Table I. Risk factors and their significance for the progression of OA after RAO in 60 hips (23 using the original technique and 37 using a modified technique)

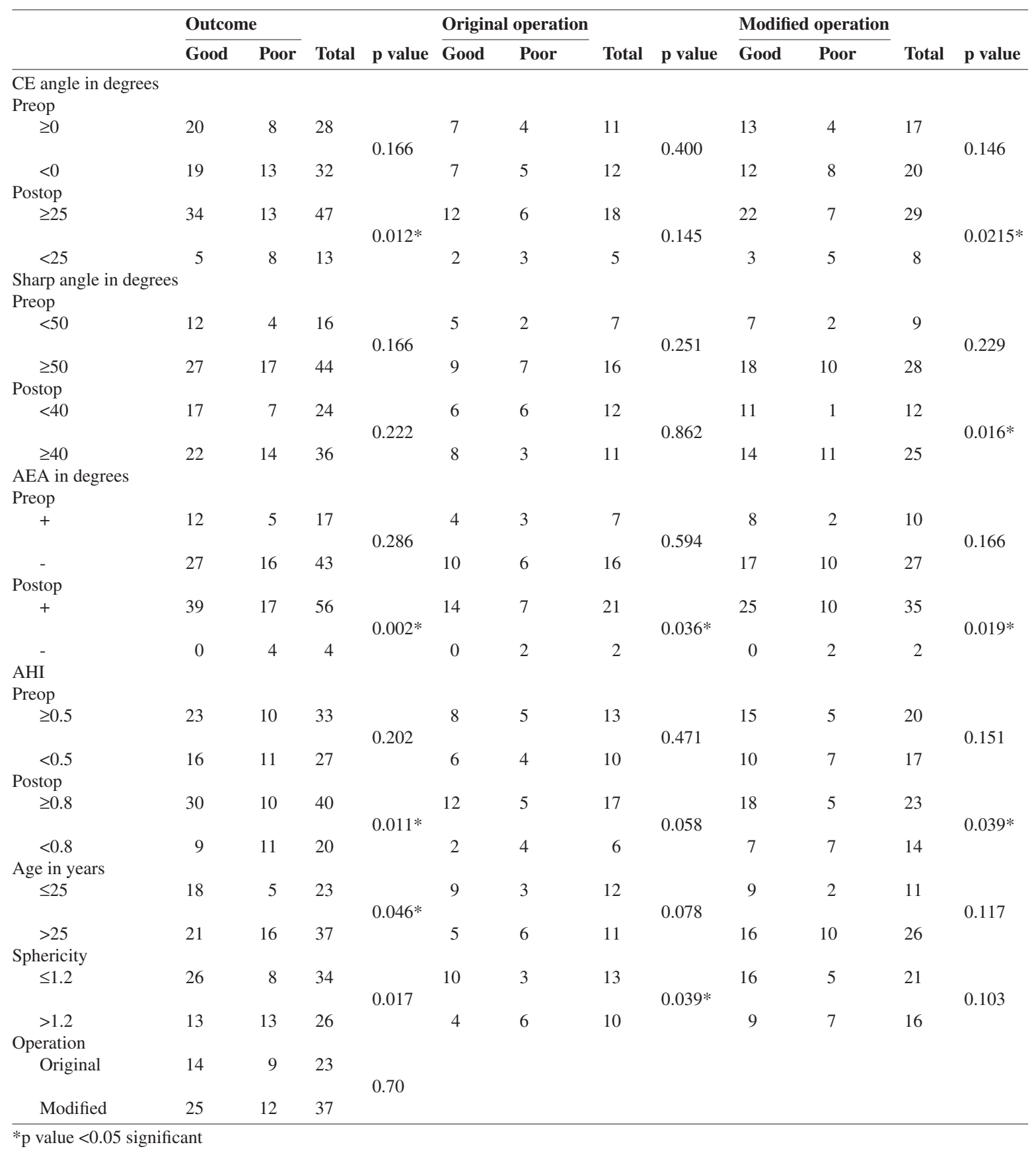

Johnson ${ }^{10}$ stated that the hip undergoes regressive and progressive remodelling in order to retain its 'physiological incongruity'. An imbalance of this remodelling with age causes destructive changes in articular cartilage. Our results certainly showed better overall outcomes in younger patients.

Our comparison between a conventional and a modified approach shows that postoperative grades are influenced by postoperative cover with the modified approach. Preoperative conditions, such as sphericity of the femoral head, influence the results with the conventional approach. Age at the time of surgery, which affects the results as a whole, did not have any special effect for either approach. A good outcome will be obtained with a modified approach, regardless of preoperative conditions, such as preoperative cover, age and sphericity of the femoral head, if postoperative cover is sufficient.

We conclude that RAO is an effective treatment, even in severe hip dysplasia, if patients are young and have little deformity of the femoral head. It is critical that sufficient acetabular cover is obtained, especially when RAO is undertaken using our modified technique. 
No benefits in any form have been received or will be received from a commercial party related directly or indirectly to the subject of this article.

\section{References}

1. Nakamura T, Yamaura M, Nakamitu S, Suzuki K. The displacement of the femoral head by rotational acetabular osteotomy: a radiographic study of 97 subluxated hips. Acta Orthop Scand 1992;63:33-6.

2. Ninomiya S, Tagawa H. Rotational acetabular osteotomy for the dysplastic hip. J Bone Joint Surg [Am] 1984;66-A:430-6.

3. Ninomiya S. Rotational acetabular osteotomy for the severely dysplastic hip in the adolescent and adult. Clin Orthop 1989;247:12737.

4. Shindo H, Igarashi H, Taneda H, Azuma H. Rotational acetabular osteotomy for severe dysplasia of the hip with a false acetabulum. J Bone Joint Surg [Br] 1996;78-B:871-7.
5. Takatori Y, Ninomiya S, Nakamura S, Morimoto S, Sasaki T. Longterm follow-up results of rotational acetabular osteotomy in painful dysplasitc hips: efficacy delaying the onset of osteoarthritis. Am J Orthop 1996;25:222-5.

6. Ueda T, Tohkura A. Evaluation of isokinetic hip strength after rotational acetabular osteotomy. Nippon Seikeigeka Gakkai Zasshi 1993;67:1105-13.

7. Wiberg G. Studies on dysplastic acetabula and congenital subluxation of the hip joint: with special reference to the complication of osteoarthritis. Acta Chir Scand 1939;83:Suppl. 58.

8. Wagner H. Osteotomies for congenital hip dislocation. In: The hip. Procs fourth open scientific meeting of The Hip Society. St Louis, USA: CV Mosby Co, 1976:45-66.

9. Matsui M, Masuhara K, Nakata K, et al. Early deterioration after modified rotational acetabular osteotomy for the dysplastic hip. J Bone Joint Surg [Br] 1997;79-B:22-4.

10. Johnson LC. Kinetics of osteoarthritis. Lab Invest 1959;8:1223. 